\title{
Some Features of Clinical Laboratory and Instrumental Research in Chronic Inflammatory Diseases of the Nose and Paranasal Sinuses in Patients with Myocarditis
}

\author{
Djuraev Abdukakhar, Djuraev Jamolbek, Akhmadaliev Nusrat, Botirov Abdurasul, Shoumarov \\ Azizkhon
}

\begin{abstract}
The issues of diagnosis and adequate treatment of rhinosinusitis $(\mathrm{RS})$ remain very relevant today, since this disease is one of the most widespread in the whole world. So, up to $15 \%$ of the adult population suffers from various forms of RS; in children this disease is even more common. Otorhinolaryngologists and primary care physicians face $R S$ in their practice on a daily basis. It should be noted that in most European countries and in America, it is the general practitioner who plays the main role in the treatment of uncomplicated forms of the disease. It is important to note that both acute and chronic RS lead to a significant decrease in the quality of life index, comparable with data on cardiological diseases and obstructive pulmonary disease.
\end{abstract}

Key words:
PNS - paranasal sinuses
ENT - ear, nose and throat
CT - computed tomography
KFK - creatine kinase
LDH - lactate dehydrogenase
CIC - circulating immunocomplex.

\section{INTRODUCTION}

Inflammatory diseases of the nose and paranasal sinuses are the most common diseases of the ear, nose and throat organs (ENT). Pollution and gas contamination of the air, its bacterial contamination, an increase in the number of respiratory viral infections and the number of inhaled allergens contribute to the growth of diseases of the nasal mucosa and paranasal sinuses. In this regard, increasing the efficiency of the diagnosis and treatment of inflammatory diseases of the nose and paranasal sinuses (PNSs) is the most important problem of modern practical otorhinolaryngology.

According to the World Health Organization (WHO), every year 40 million people suffer from infectious diseases, of which $90 \%$ correspond to influenza and acute respiratory diseases (ARD). Scientists confirm that about $80 \%$ of patients with various acute infectious diseases can experience dysfunction of the cardiovascular system. Many of them are a functional reaction of the body and they can recover without specific treatment. But in some cases of a

Revised Manuscript Received on October 15, 2019. Uzbekistan.(Email: abdukahar.djuraev@ @ tsdi.uz)

Djuraev Jamolbek, Tashkent Medical Academy, Uzbekistan.(Email jamolbek.djuraev@tma.uz)

Akhmadaliev Nusrat, Tashkent State Dental Institute, Uzbekistan.(Email: nusrat.akhmadaliev@tsdi.uz)

Botirov Abdurasul, Tashkent Medical Academy, Uzbekistan.(Email: abdurasul.botirov@tma.uz) Uzbekistan.(Email: azizkhon.shaumarov@tma.uz).
Djuraev Abdukakhar, Tashkent State Dental Institute,

Shoumarov Azizkhon, Tashkent Medical Academy,

certain part of patients, cardiac arrhythmias are observed and this process continues for a long time, and in some cases due to the development of heart failure it ends in death.

Most scientists believe that from 1 to $5 \%$ of all patients with acute respiratory infections, including influenza, have signs of infectious myocarditis. In the pathogenesis of myocardial infectious lesions, not only the causative agents of the underlying disease themselves, but also the regular components of the infectious-inflammatory process: intoxication, discirculatory, dysmetabolic and immune disorders are of direct importance.

To date, there is a large amount of data suggesting that autoimmune damage is the second phase of the infectiousinflammatory process in the myocardium, or inflammatory cardiomyopathy.

The most important form of communication between the body and the external environment, which does not stop throughout a person's life, is communication through the respiratory system. Nasal breathing is a normal physiological event, and its violation can cause functional and morphological changes in the life and structure of the most important organs and systems of the body, which occur as a result of a slowdown in metabolic processes, a decrease in the bioelectric potential of the cell, and the appearance of a state of energy deficiency due to oxygen deficiency, impaired microcirculation, as well as weakening of the ciliary and epithelial functions. Turning off nasal breathing leads to obstruction of the venous outflow, which causes an increase in $\mathrm{pCO} 2$ blood pressure and changes in the coronary vessels. These circumstances explain the increased attention of rhinologists and cardiologists to issues related to the correction of nasal breathing in various pathologies of the nasal cavity.

In the world, to achieve optimization of the treatment of patients with diseases of the nose and paranasal sinuses, research is being conducted, a study aimed at studying the degree of occurrence of diseases of the nose and paranasal sinuses in patients with myocarditis. In patients with myocarditis with diseases of the nose and paranasal sinuses there is no justification for a comparative assessment of clinical, laboratory, functional, instrumental and microbiological studies, the effect of the mechanism of action of nasal congestion on patients with diseases with the cardiovascular system, the development of an algorithm for the diagnosis and treatment of patients with myocarditis with diseases of the nose and around the sinuses. Of 
particular importance are the development of a system aimed at strengthening the health status of patients with myocarditis with diseases of the nose and nasal sinuses.

\section{MATERIAL AND METHODS}

The aim of this study was to study the characteristics of indicators of clinical, laboratory and instrumental studies in chronic inflammatory diseases of the nose and paranasal sinuses in patients with myocarditis.

The material of this study was 150 patients with myocarditis who were hospitalized in the Republican Specialized Center of Cardiology from 2016 to 2019. The age of patients was from 18 years to 70 years. Patients were divided into 3 groups. The first group consisted of 80 patients with chronic inflammatory diseases of the nose and paranasal sinuses with myocarditis. The second group consisted of 50 patients with chronic inflammatory diseases of the nose and paranasal sinuses. The third group consisted of 20 patients with myocarditis. A thorough history was collected from all patients and a comprehensive clinicallaboratory, endoscopic, functional, radiographic and microbiological examination was carried out. Statistical data processing was performed using Microsoft Office 2016.

Each patient's thematic map includes the results of clinical and functional studies:

- The study of complaints and medical history data;

- Traditional examination of ENT organs;

- Endoscopic examination of the nasal cavity and nasopharynx;

- Computed tomography of the paranasal sinuses (CT-PNS);

- Assessment of ciliary functional abilities. epithelium (determination of the time of mucociliary transport, activity of the absorption and excretory functions of the nasal mucosa).

- $\quad$ Study of local immunological status;

- Bacteriological examination of the mucous membrane of the nasal cavity and puncture of the maxillary sinuses;

- Allergological tests.

When collecting an anamnesis, the timing of the onset of the disease was clarified, the timing of relapses, their relationship with infectious diseases, diseases of the respiratory system, as well as the presence of a burdened allergic history were taken into account.

Examination of ENT organs included: anterior and posterior rhinoscopy, oropharyngoscopy, otoscopy, indirect laryngoscopy, endoscopic examination of the nasal cavity and pharynx.

For endoscopic examinations, we used rigid endoscopes from Karl Storz (Germany) with a diameter of $4.0 \mathrm{~mm}$ with end and side optics of 0,30 and 70 degrees.

Endoscopic examination of the nasal cavity was carried out by us according to the method of Messerldinger W. in the patient's sitting position, before and after anemization of the nasal mucosa before surgical correction, as well as in all subsequent patient visits (on days 14, 28, after 3 months, 6 months, 1 year and then every year for 3 years). All sections of the nasal cavity were examined sequentially, starting from the vestibule and the nasal valve. Particular attention was paid to anomalies of the middle nasal passage and nasopharynx. The middle nasal concha was examined. Inspection was carried out, starting from its posterior end, with the reverse movement of the endoscope. One could observe the paradoxical curvature of the middle nasal concha, which could lead to impaired ventilation of the paranasal sinuses.

Clinically, it is important to assess the condition of the ostiomeatal complex located in the anterior middle nasal passage. This name was given to the front-ethmoid complex in 1965 by Naumann, which determined its importance in the development of diseases of the frontal and maxillary sinuses. Its components: the front end of the middle shell, the hook-shaped process, the cells of the nasal tubercle, lunate fissure, frontal pocket, ethmoid bulla and lateral sinus. Since all natural openings of the maxillary, frontal and anterior cells of the ethmoid bone are concentrated in the ostiomeatal complex, it is key in the pathogenesis of acute and chronic sinusitis.

The last stage is inspected the upper nasal passage. Here we can examine the openings of the posterior sinuses of the ethmoid bone, the sphenoethmoid pocket and the anastomosis of the sphenoid sinus, which is located above the choana between the nasal septum and the superior nasal concha.

With the help of a complete endoscopic examination, it is possible to more accurately determine the causes of acute relapsing diseases of the nasal cavity and paranasal sinuses and factors that aggravate the development of the inflammatory process in the nasal cavity. Accordingly, the selection of a method for treating a disease is facilitated.

\section{METHODS OF STUDYING THE FUNCTIONAL ABILITIES OF THE CILIATED EPITHELIUM.}

The study of mucociliary transport (MCT), excretory and absorption functions of the nasal mucosa was carried out using polymer soluble films containing saccharin and methylene blue on both sides. The rate of dissolution, movement and absorption of the indicator in different halves of the nose and in different parts of the mucous membrane is different. There are fairly constant ways of moving the gel, but the speed of movement periodically changes. The movement of mucus in the nasal cavity in several main directions indicates that at a certain moment one group of cells carries out active secretion transport, and the other is at rest. After a certain period of time, the degree of their activity changes.

Before the study, anemization of the nasal mucosa was not carried out and no drugs were administered. We placed polymer films with a size of 1 by $0.3 \mathrm{~cm}$ on the mucous membrane of the medial surface of the lower nasal turbinate, retreating $1-1.5 \mathrm{~cm}$ from its front end. During the study, the patient was asked not to force breathing, not to cough, not to blow your nose and not to sneeze, not to eat, to swallow movements once a minute, not to throw back your head sharply, to be in a sitting position. During the study, we performed a back rhinoscopy once every 2-3 minutes.

Blue Eyes Intelligence Engineering 
The transport function was evaluated by the time through which the mucus tinted with methylene blue appeared in the nasopharynx and a sweetish taste appeared in the mouth.

The secretory activity of numerous mucous and serous glands, which are located in the own layer of the nasal mucosa, as well as atrial epithelial cells, is the basis of the excretory function. The intensity of excretory function is determined by the amount of secretion produced by the mucous membrane of the nasal cavity, and the period of time from the moment the film is applied to the surface of the mucous membrane until it is completely dissolved.

We evaluated the absorption capacity of the mucous membrane of the nasal cavity by the degree of its staining at the location of the polymer film. The more pronounced the inhibition of the motor activity of the cilia, the stronger the staining of the mucous membrane with methylene blue, that is, the best conditions for absorption are created. The absorption capacity of the mucous membrane increases dramatically during atrophic processes, with inhibition of the movement of the cilia with various medications. The test was performed for patients before surgery, 14 days after surgery, 1 month, 3 months, 0.5 years and 1 year after surgery.

In the case of a sharp slowdown in mucociliary transport, strong staining of the mucous membrane of the nasal cavity at the marking site can be seen even with anterior rhinoscopy (pic. 1). If repeated tests confirm the immobility of the labels introduced into the nasal cavity, it can be conclusively stated that this patient has a mucus transport disorder, although only electron microscopy of biopsy samples of the mucous membrane of the nasal cavity can establish the presence of structural pathology of the cilia.

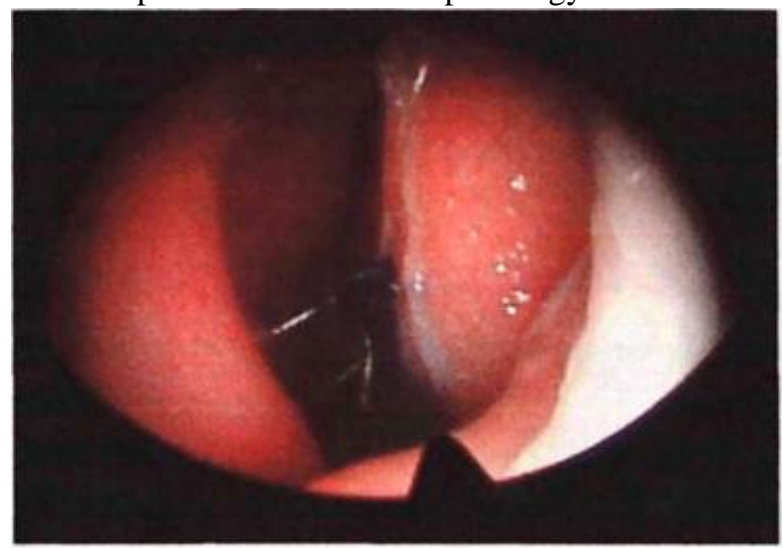

Pic. l. The study of mucociliary transport using polymer films stained with methylene blue. The film is installed on the lower nasal concha ( 20 minutes from the start of the study - the film does not dissolve, does not advance, does not absorb, which indicates a violation of the patient's MCT with a curved nasal septum and vasomotor rhinitis.).

Bacteriological examination of the secretion of the nasal cavity and maxillary sinuses between relapses of the disease, and at the time of relapse - punctate from the affected sinus and from the nasal cavity.

Material was taken from the nasal cavity (between relapses) for this study using anterior rhinoscopy from under the middle nasal concha with a sterile swab on a metal stick, which was immediately placed in a dry sterile tube and immediately plated on a Petri dish. At the time of relapse, the maxillary sinus was punctured with a Kulikovsky needle, the contents of the sinus were aspirated with a sterile disposable syringe. For delivery to the laboratory, sterile tubes with Amies W / CH preservation medium (Italy) were used. Microbiological methods included the study of the species composition of the microflora of the nose on solid nutrient media; After species identification of the seeded microflora, its sensitivity to antibiotics of various groups was studied by agar diffusion with standard indicator discs. For the cultivation of streptococcus and hemophilic bacillus cultures, microaerophilic conditions were created using $10 \%$ $\mathrm{CO} 2$.

We also took smears on the flora from the mucous membrane of the nasal cavity in patients and in control groups 1 year after the end of treatment to determine how its character changes after our intervention.

Laboratory and functional diagnostic studies were carried out on days 2-5 of the illness and on days 26-30 of hospital stay against the background of the absence of clinical signs of the disease. Blood sampling for laboratory tests was carried out in the morning on an empty stomach also on days 2-5 of the disease and on days 26-28 in hospital. The values of the indicators were determined by standardized laboratory methods. Blood biochemical parameters (total protein, albumin, globulin, AST, ALT, LDH, CPK, urea, creatinine, etc.) were determined using a Hitachi-714 biochemistry analyzer (Japan) with reagents from Boehringer Mannheim (Austria). The concentrations of $\operatorname{IgA}$, IgM, IgG in blood serum were determined by radial immunodiffusion, the content of serum CIC by the precipitation method using a solution of 3.5 polyethylene glycol. Factors of cellular immunity (T-, B-lymphocytes, subpopulations of T-lymphocytes in peripheral blood were determined by the method of rosette formation. The inhibition of leukocyte migration was carried out with phytohemagglutinin. A total of 36 indicators of laboratory methods of research at the beginning of an infectious disease and after 4 weeks of hospital stay were analyzed. Of these, indicators of values of a general clinical blood test - 7 , biochemical blood tests -10 , indicators of immunological tests -19 .

\section{RESULTS AND DISCUSSION}

Rhinosinusitis is an inflammation of the nasal mucosa and paranasal sinuses (PNS), almost always caused by stagnation of the secret and a violation of aeration of the PNS. The starting point in the development of rhinosinusitis is usually a viral infection [4].

In recent years, there has been a significant increase in the number of diseases of the nose and paranasal sinuses both in absolute numbers and as a percentage of the total number of ear, nose and throat (ENT) diseases - [1,5]. According to some authors, over the past decade, the number of cases of sinusitis per 1000 people has increased from 4.6 to 12.2. During this period, the number of patients with diseases of the nose and paranasal sinuses increased annually by $1.5-$ $2 \%$ and reached $52.7 \%$ [3.8]. 
Factors that violate the patency of the natural openings of the paranasal sinuses and the mechanisms of their aeration and cleansing include abnormalities of the development of the nasal concha, curvature of the nasal septum, and deformation of the nasal valve, whose functions have not yet been fully studied $[2,3,9,12]$. The rendered pathological action creates the conditions for blockade of the ostiomeatal complex, and subsequently the development of the inflammatory process in the paranasal sinuses $[4,5,6,7,9,11]$. In conditions of stagnation of the secret and a decrease in the partial pressure of oxygen in the PNS, favorable conditions are created for the development of a bacterial infection $[12,13,14,15]$.

The number of rhinosinusogenic orbital and intracranial complications, often leading to disability or death of the patient, has at least not decreased recently $[16,17]$.

According to the authors, rhinosinusitis significantly reduces the quality of life of people. When studying the general health status, patients with rhinosinusitis had significantly lower indicators of pain sensitivity and social activity, sleep disturbance, emotional state deteriorated (compared with patients with coronary insufficiency and chronic obstructive pulmonary diseases [19,20].

Annually, 40 million people become infected with infectious diseases in the world, $90 \%$ of which are influenza and acute respiratory infections. Over the past 10-20 years, the incidence of myocarditis and pericarditis has increased significantly [18]. Non-coronarogenic myocardial diseases account for $7-9 \%$ of all diseases of the cardiovascular system $[10,21]$. It has been proven that there is a direct connection between influenza and acute respiratory infections with subsequently developing chronic therapeutic pathology and serious somatic complications [22]. It is considered possible to develop primary heart damage in acute infectious diseases [4,6,12], myocarditis and dilated cardiomyopathy in patients who have had a viral infection $[1,9,16]$. Of particular relevance is the study of the role of inflammatory diseases of the ENT organs in the occurrence of heart pathology, which very often are the cause of dystrophy of the heart muscle [2], myocarditis and rheumatism $[6,8]$.

Impaired cardiovascular function can occur in approximately $80 \%$ of patients with various acute infectious diseases [5,7]. Most of them are a natural functional response of the body, complete independently, without additional special treatment. But some children develop rhythm disturbances, heart failure, often with a prolonged protracted course, and sometimes with a risk of death.

Currently, it is known that any of the known pathogens can cause damage to the myocardium, including myocarditis [11]. Often the etiological factor is viruses that can directly interact with cardiomyocytes, which leads to cell apoptosis [17].

To detail the nature of the lesion of the nasal cavity and paranasal sinuses allow special examination methods, which have been included in the arsenal of ENT specialists in recent years. This is an instrumental endoscopic study, with which you can objectively assess the degree of violation of nasal breathing $[6,7,8,10]$.

Endoscopy of the nasal cavity is the most reliable method for examining the nasal cavity, the condition of the paranasal sinuses and their natural anastomoses and plays a dominant role in the diagnosis, an objective assessment of the effectiveness of the treatment and the choice of the best option for surgical intervention $[4,12]$.

Using an endoscope, it is possible to sequentially examine all parts of the nasal cavity, starting from the vestibule and the nasal valve. Pay attention to the color of the mucous membrane, the presence and nature of the discharge, evaluate the size of the nasal concha, as well as the condition of the pharyngeal tonsil and the mouth of the auditory tubes [7,11]. Endoscopy helps identify nasopharyngeal cysts, as well as confirm the diagnosis of Tornwald bag [16].

The solution to the problem of timely detection of diseases of ENT organs in patients with myocarditis and the development of effective treatment methods are associated with an in-depth study of the pathogenetic mechanisms of their development and the search for highly informative diagnostic criteria. Changing economic conditions and the introduction of new medical technologies into practice necessitated a medical and economic justification of the recommended diagnostic methods and treatment and rehabilitation measures.

A study of the anamnesis showed that chronic inflammatory diseases of the nose and paranasal sinuses in patients with myocarditis were more likely to occur at the age of 31-50 years. No gender difference was observed.

Patients with diseases of the nose and paranasal sinuses most often had difficulty breathing $(81.5 \%)$, discharge from the nose $(30.0 \%)$, pain in the projection of the paranasal sinuses $(24.5 \%)$, pain in the area of the heart $(80,5 \%)$.

The main complaints presented by patients were difficulty in nasal breathing (92.5\%), discharge from the nose $(78.4 \%)$, impaired sense of smell $(22.2 \%)$, low-grade fever $(36.4 \%)$, general weakness $(42.5 \%)$. Often patients noted pain $(78.4 \%)$ in the maxillary region. Computed tomograms in all patients revealed various combinations of the paranasal sinuses involved in the pathological process. In 31 patients, isolated lesions of the maxillary sinuses were detected, in 29 - lesions of the maxillary and ethmoid sinuses, in 14 - lesions of the ethmoid and frontal sinuses, 6 patients revealed lesions of the maxillary, ethmoid and main sinuses. In 45 patients, a curvature of the nasal septum was revealed, in 7 - polyps, in 11 - hypertrophy of the ethmoid bulla, in 17 - hypertrophy of the lower nasal concha (pic. 2,3). 


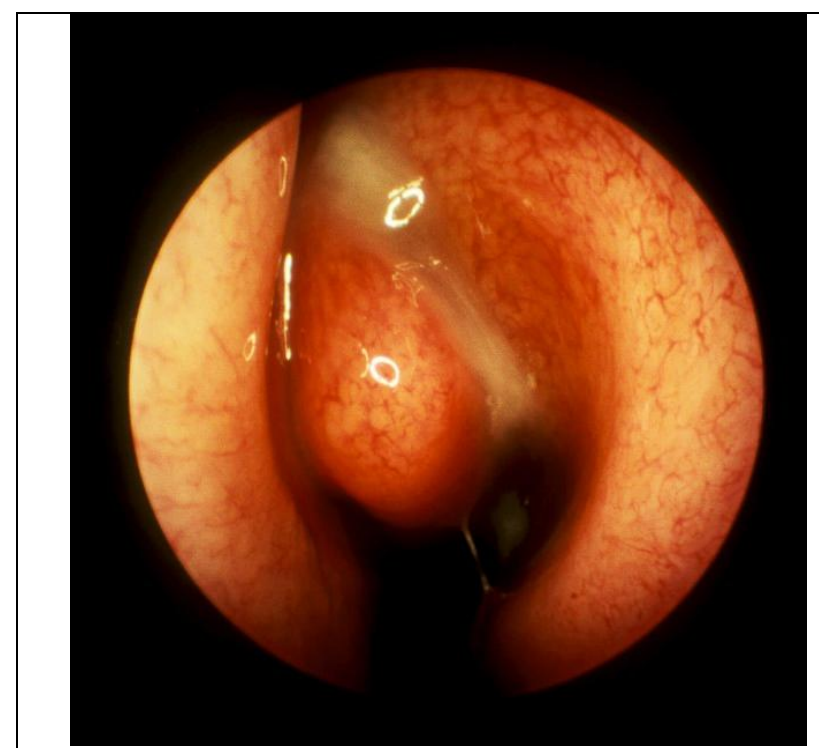

Pic. 2. With endoscopy of the nose, purulent discharge in the middle nasal passage on the left is noted

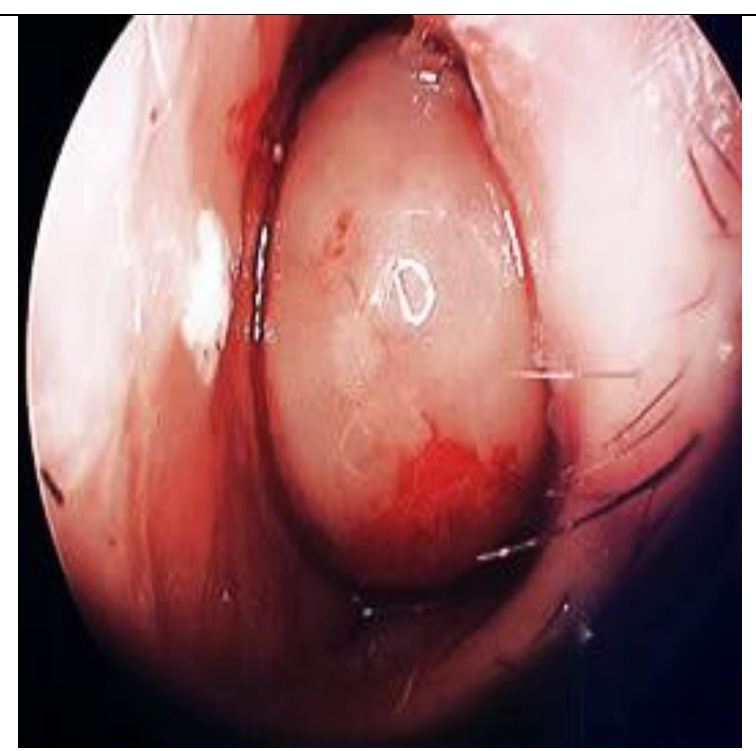

Pic. 3. With endoscopy of the nose, an increase in the lower nasal concha on the left
As indicated above, an endoscopic examination of the nasal cavity was performed before and after treatment (pictures 1,2). The results of endoscopic studies showed that in all patients hyperemia and swelling of the nasal mucosa was determined, in 62 - pathological discharge in the nose, in 45 - curvature of the nasal septum, in 7 - polyps in the middle nasal passage, in 13 - pathology of the middle nasal concha, in 5 - hypertrophy of the hook process, in 18 hypertrophy of the inferior nasal concha, in 10 patients hypertrophy of the ethmoid bulla.

According to endoscopic studies, in the 1st observation group it was revealed: hyperemia and swelling of the nasal mucosa in 32 patients, nasal septum curvature - 21 patients, lower turbinate hypertrophy - 24 patients, pathological discharge - 12 patients, trellised bulla hypertrophy in 2 patients, hypertrophy of the hooked process - 2 patients. In the second group, hyperemia and swelling of the mucous membrane of the nasal cavity was observed in 24 patients, deformation of the nasal septum - 24 patients, hypertrophy of the inferior nasal concha - 18 patients, pathological discharge -8 patients, hypertrophy of the ethmoid bulla in the 1 st patient, hypertrophy of the hook process - in 3 patients.

With endoscopy in the absence of nasal polyps given 0 ; polyps that do not extend beyond the middle of the nasal concha and for visualization requiring endoscopic examination are given 1; polyps that extend beyond the middle of the nasal concha and visible through the nasal mirror are given 2 ; category 3 is given to massive polyps that cover the nasal cavity.

Radiography and computed tomography (CT) scan of the paranasal sinuses revealed hypertrophy of the inferior nasal concha in combination with deformation of the nasal septum in 13 patients of the 1 st group and 25 patients of the 2 nd group. Hypertrophy of the middle nasal concha in combination with deformation (mixed rhinopathologes) of the nasal septum was observed in 11 patients of the 1 st group and 18 patients of the 2nd group, isolated hypertrophy of the lower nasal concha was detected in 12 patients of the 1st group and 7 patients of the 2nd group. Hypertrophy of the inferior nasal concha in combination with hypertrophic ethmoid bulla and deformation of the nasal septum is observed in 2 patients of group 1 and group 2, respectively, hypertrophy of the middle nasal concha in combination with hypertrophy of the hook process was revealed in the $1 \mathrm{st}$ patient of the 1 st and 2 nd group respectively.

The study of mucociliary transport function was carried out using a saccharin test in 130 patients with diseases of the nose and paranasal sinuses. For comparison, 20 relatively healthy recipients were selected who did not have a history of diseases of the nose and paranasal sinuses. In the comparison group, after the saccharin was introduced into the nasal cavity, the appearance of a sweet taste in the oral cavity occurred within 9-19 minutes.

In patients with diseases of the nose and paranasal sinuses against the background of myocarditis, the average mass of balls was $58.4 \pm 0.8 \mathrm{mg}$, in patients only with diseases of the nose and paranasal sinuses $-55.7 \pm 0.8 \mathrm{mg}$. Studies have shown that both the 1st and 2nd observation groups significantly impaired the transport function of the nasal mucosa, there is a change in $\mathrm{pH}$, increased absorption time, increased excretory function led to increased secretion. Indicators of excretory function or secretory activity of the nasal mucosa in patients with chronic rhinosinusitis are significantly increased. This indicates the overproduction of the mucous and goblet glands, as a result of the inhibition of the activity of the ciliary epithelium lysozyme.

To identify violations of nasal patency, the rhinopneumotachometry method was used, the results of which were used as qualitative and quantitative indicators.

When conducting studies characterizing the functional state of the nasal cavity, it was found that in 1 and 2 groups of observations, a decrease in respiratory function, i.e. nasal patency (FEF75 / MOS75) was 31.4-0.9 (p <0.001 compared with the control). There is also a lengthening of the transport function time (with an average of $39.7 \pm$ 0.91 minutes $) \quad(\mathrm{p} \quad<0.001$

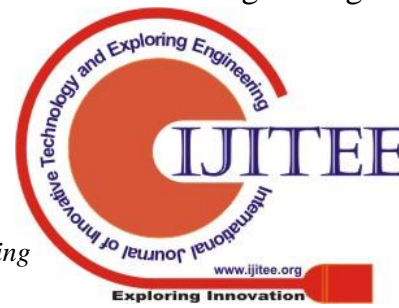



$8.2 \pm 0.01$ ) (with $\mathrm{p}<0.001$ ), increased absorption time (average $91.4 \pm 6.9$ minutes) (at $\mathrm{p}<0.01$ ), increased excretory function (on average, $65.1 \pm 0.9 \mathrm{mg}$, at $\mathrm{p}<0.001$ compared with the control). In both groups, the observed dysfunction of the nasal mucosa led to a decrease in the quality of life of patients. Therefore, when choosing a treatment strategy for patients, of course, this situation should be taken into account.

When studying the microflora of the nasal mucosa in patients of groups 1 and 2, the prevalence of gram-positive strains (Staphylococcus epidermidis, Streptococcus pneumoniae, Sreptococcus viridans, Staphylococcus aureus) was revealed. A general analysis of the results revealed that the strain Staphylococcus epidermidis - mainly prevails (47 patients $-36.1 \%$ of all cases of observation). In $24.6 \%$ of cases, Staphylococcus aureus is determined (32 patients). The strains of Streptococcus viridans are the least encountered - 12 patients (9.2\%), Pseudomonas aerogenosa - 11 patients (8.5\%), Hemophilus influenzae - only in 2 cases $(1.5 \%)$. Of particular importance was the presence of beta-lactamase producers with a wide spectrum of action, as well as a representative of enzyme-forming bacteria (Pseudomonas aeruginosa-8 cases $(6.50 \%)$. Also in addition to the above strains were identified: Proteus vulgaris in 7 patients $(5.69 \%)$, Citrobacter freundii - 4 patients $(3.25 \%)$, E. coli - in 2 patients $(1.62 \%)$.

Biochemical blood tests were performed in the laboratory studied cardiospecific enzymes that act as catalysts for the breakdown of creatine phosphate in the heart muscle, the transfer of amino groups to keto acids from amino acids, an increase in which indicates damage to the myocardium or its necrosis, and the activity of the creatine kinase-MV isoenzyme (KFK-MV) was also determined. As our studies have shown, the level of indicators KFK-MV and lactate dehydrogenase (LDH) is significantly increased in the blood of patients of the 1st and 3rd observation groups (Tabl. 1)

Table 1. Data from biochemical blood tests: indicators of the content of cardiospecific enzymes in the blood and indicators of the level of CIC

\begin{tabular}{|c|c|c|c|c|}
\hline $\begin{array}{l}\text { Indicato } \\
\text { rs }\end{array}$ & $\begin{array}{l}1^{\text {st }} \text { group } \\
n=80\end{array}$ & $\begin{array}{l}2^{\text {nd }} \text { group } \\
\mathbf{n}=\mathbf{5 0}\end{array}$ & $\begin{array}{l}3^{\text {rd }} \text { group } \\
\mathbf{n}=\mathbf{2 0}\end{array}$ & $\begin{array}{l}\text { Control } \\
\text { group } \\
\mathbf{n = 2 0}\end{array}$ \\
\hline $\begin{array}{l}\text { CPK } \\
(\mu \mathrm{mol} \mathrm{g} / \\
1)\end{array}$ & $\begin{array}{l}7,68 \pm 0,02 \\
*\end{array}$ & $\begin{array}{l}4,12 \pm 0,03 \\
*\end{array}$ & $\begin{array}{l}6,54 \pm 0,05 \\
*\end{array}$ & $\begin{array}{l}2,87 \pm 0,7 \\
5\end{array}$ \\
\hline $\begin{array}{l}\mathrm{LDH} \\
(\mu \mathrm{mol} \mathrm{g} / \\
1)\end{array}$ & $\begin{array}{l}6,41 \pm 0,04 \\
*\end{array}$ & $5,6 \pm 0,04$ & $\begin{array}{l}6,01 \pm 0,02 \\
*\end{array}$ & $4,9 \pm 0,64$ \\
\hline \multicolumn{5}{|c|}{ CIC (ng / ml): } \\
\hline Large & $\begin{array}{l}18,4 \pm 0,94 \\
*\end{array}$ & $11,6 \pm 0,24$ & $14,9 \pm 0,45$ & $\begin{array}{l}10,2 \pm 0,3 \\
3\end{array}$ \\
\hline Small & $\begin{array}{l}23,1 \pm 0,16 \\
*\end{array}$ & $9,7 \pm 0,042$ & $\begin{array}{l}19,7 \pm 0,35 \\
*\end{array}$ & $9,5 \pm 0,37$ \\
\hline
\end{tabular}

compared with the control), an increase in $\mathrm{pH}$ (on average of the Republican Specialized Center for Cardiology. We

Note: * $-\mathrm{P}<0.05$, the reliability of the results in relation to the control values

When determining the level of the circulating immunocomplex (CIC), a significant increase in indicators was revealed in both 1 and 2 groups, an increase in indicators was observed already from the first days of the disease and remains up to 15 days of observation. So, in the 1 st group the increase was $64 \%$, in the 3rd group - $53 \%$ and in the second group $42 \%$ in relation to the maximum threshold indicator - 77 units (1-table).

Based on the results obtained, it is fashionable to conclude that the observed development of the immunopathological process can be divided into a number of stages. At the beginning of the disease, when the pathogen enters the body, the observed characteristic reactions are determined by cellular and humoral defense factors. As non-specific manifestations of inflammation, one can note an increase in the phagocytic activity of neutrophils, leukocytosis, an increase in the parameters of EST, fibrinogen, aminotransferase, CRP, ASLO, sialic acid. The involvement of macrophages in the immune process becomes the trigger mechanism for the activation of $\mathrm{T}$ and $\mathrm{B}$ lymphocytes. The conversion of B lymphocytes into plasma cells enhances secretion. Class $\mathrm{M}$ and $\mathrm{B}$ immunoglobulins are complement activators and are involved in the breakdown of antigens. However, in patients of the 3rd group, despite clinical recovery, signs of an ongoing immunopathological reaction and myocardial damage persist.

In order to study the degree of hypoxia in patients with diseases of the nose and paranasal sinuses with obstruction of the nasal cavity, we determined the level of partial pressure $\mathrm{pO} 2$ and pCO2 (talb. 2).

Table 2. Blood pO2 and pCO2 levels

\begin{tabular}{|l|l|l|l|l|}
\hline $\begin{array}{l}\text { Indicator } \\
\mathrm{s}\end{array}$ & $\begin{array}{l}1^{\text {st }} \\
\mathrm{n}=80\end{array}$ & $\begin{array}{l}2^{\text {nd }} \text { group } \\
\mathrm{n}=50\end{array}$ & $\begin{array}{l}3^{\text {rd }} \text { group } \\
\mathrm{n}=20\end{array}$ & $\begin{array}{l}\text { Norm } \\
\mathrm{a} \\
\mathrm{n}=20\end{array}$ \\
\hline $\mathrm{pO}_{2}$ & $\begin{array}{l}26,4 \pm 8,2 \\
*\end{array}$ & $\begin{array}{l}24,6 \pm 9,5^{*} \\
*\end{array}$ & $32,6 \pm 3,8$ & 40,2 \\
$\mathrm{pCO}_{2}$ & $\begin{array}{l}47,7 \pm 1,9 \\
*\end{array}$ & $\begin{array}{l}50,2 \pm 3,7 * \\
*\end{array}$ & $\begin{array}{l}42,6 \pm 1,6 \\
9\end{array}$ & 45,1 \\
\hline
\end{tabular}

Note: $*-p<0.05$ reliably in relation to the 1 st and 2 nd group of patients, $* *-p<0.05$ reliably in relation to the 1 st and 2 nd group of patients.

When studying the level of partial pressure pO2 and pCO2 in patients with obstruction of the nasal cavity, a sharp change in gas exchange processes was revealed.

The results of the studies showed that in the blood of patients of 1-2 groups with severe obstruction of the nasal cavity against the background of inflammatory processes in the mucous cavity and paranasal sinuses, an increase in the level of CO2 from $42.6 \mathrm{~mm} \mathrm{Hg}$ is noted. up to $50.2 \mathrm{mmHg}$ In contrast, the level of $\mathrm{O} 2$ in the blood decreases markedly from $32.6 \mathrm{~mm} \mathrm{Hg}$. up to $24.6 \mathrm{mmHg}$ A detected violation of gas exchange processes, the presence of hypoxia is observed 
in patients of 1-2 observation groups, however, it should be noted that the severity of hypoxia depends on the deformation of intranasal structures joining the existing pathology of the nose and paranasal sinuses.

In the postoperative period, all patients were prescribed rinsing of the nasal cavity by moving drugs according to the Proets method. Among the drugs, we included the most sensitive to this type of infection in the nose. Topical use of the drug Nazonex in a dose of $125 \mathrm{mcg}$ in each half of the nose 2 times a day for 7 days. The criteria for the effectiveness of treatment were: positive dynamics in diagnostic endoscopy of the nasal cavity, as well as an analysis of outpatient cards and a subjective assessment of their condition by the patient himself.

In the endoscopic examination, the following scoring system is used to diagnose nasal polyps (tab. 3 ).

Table 3. Scoring system

\begin{tabular}{|l|l|l|l|l|l|}
\hline Character & Basic & $\begin{array}{l}3 \\
\text { months }\end{array}$ & $\begin{array}{l}6 \\
\text { months }\end{array}$ & $\begin{array}{l}1 \\
\text { year }\end{array}$ & \begin{tabular}{l} 
year \\
\hline $\begin{array}{l}\text { Polyp on the } \\
\text { left }(0,1,2,3)\end{array}$
\end{tabular} \\
\hline $\begin{array}{l}\text { Polyp on the } \\
\text { right } \\
(0,1,2,3)\end{array}$ & & & & & \\
\hline $\begin{array}{l}\text { Edema on } \\
\text { the left } \\
(0,1,2) \text { on }\end{array}$ & & & & & \\
\hline $\begin{array}{l}\text { Edema right } \\
\text { the } \\
(0,1,2)\end{array}$ & & & & & \\
\hline $\begin{array}{l}\text { Discharge, } \\
\text { left }(0,1,2)\end{array}$ & & & & & \\
\hline $\begin{array}{l}\text { Discharge, } \\
\text { right }(0,1,2)\end{array}$ & & & & & \\
\hline Total points & & & & & \\
\hline
\end{tabular}

The observation period ranged from 6 to 24 months. In none of the cases were complications and side effects observed for topical application of bioparox.

A good result was evaluated at 0-6 points, a satisfactory result at 7-10 points, and an unsatisfactory result at 11-14 points.

The treatment results determined that a good result corresponding to 0-6 points was observed in 94 patients $(62,7 \%)$, satisfactory, corresponding to $7-10$ points - in 43 (28,6\%), unsatisfactory, corresponding to $11-14$ points -13 patients $(8,7 \%)$.

Of the total number of patients with difficulty in nasal breathing caused by various etiological factors, we selected patients and underwent surgery to eliminate them.

At the same time, we focused on the severity of myocarditis, the age of patients, complaints of difficulty in nasal breathing, and clinical laboratory data. However, in the presence of serious concomitant pathology, especially in elderly patients, initially the surgical intervention was limited to consultative methods, or minimally invasive treatment methods.

Studies indicate a pronounced course of diseases of the nose and paranasal sinuses in patients with myocarditis, in which the function of the nasal mucosa decreases, which also affects the course of myocarditis.

\section{CONCLUSIONS}

1. The relationship between diseases of the nose and paranasal sinuses and the course of myocarditis was revealed; it manifests itself more in patients during recurrence of myocarditis. In $25 \%$ of patients with myocarditis, vasomotor rhinitis is observed, in $53.75 \%$ of cases chronic rhinosinusitis, nasal septum curvature in $40 \%$ of cases, hypertrophic rhinitis - 10\%, myxrinopathology $35 \%$ of patients;

2. Clinical and functional studies in patients of the $1 \mathrm{st}$ group with diseases of the nose and paranasal sinuses against the background of myocarditis showed pronounced disturbances in transport function, $\mathrm{pH}$ changes, absorption and excretion processes, so mucociliary clearance was 25.4 minutes, absorption time 76.9 minutes, excretion - 52.3 minutes;

3. The use of endoscopy meets the requirements of modern otorhinolaryngology, is timely and indispensable in the diagnosis and treatment of chronic inflammatory diseases of the nose and paranasal sinuses. The use of endoscopic methods in the treatment of patients with chronic inflammatory diseases of the nose and paranasal sinuses can reduce the number of relapses, which favorably affects the quality of life of our patients;

4. Biochemical blood tests of patients of the 1st group showed significant changes in indicators - a decrease in pO2 level against the background of an increase in $\mathrm{pCO} 2$, an increase in $\mathrm{CPK}, \mathrm{LDH}$, large and small CICs (in patients of the 1 st group pCO2 it was $49.9 \mathrm{mmol} / \mathrm{l}$, which exceeds the average by $13,1 \%(\mathrm{p}<0.05)$; decrease in the level of $\mathrm{O} 2$ to $32.6 \mathrm{mmol} / \mathrm{L}$, on average by $50.3 \%$ ( $\mathrm{p}<0.05)$.

\section{LITERATURE}

1. Bachert C., Tomassen P. Endotype-Driven Approach for Chronic Rhinosinusitis //Implementing Precision Medicine in Best Practices of Chronic Airway Diseases. Academic Press, $2019 . \quad$ - ?. 51-57. https://doi.org/10.1016/B978-0-12-813471-9.00013-X

2. Bengtsson $\mathrm{C}$. et al. Incident Chronic Rhinosinusitis Is Associated With Impaired Sleep Quality: Results of the RHINE Study //Journal of Clinical Sleep Medicine. 2019 . - ?. 15 . - ? 06 . 06 ?. 899-905. http://dx.doi.org/10.5664/jcsm.7846

3. Bergmark R.W., Pynnonen M. Diagnosis and First-Line Treatment of Chronic Sinusitis. JAMA. 2017 Dec 19;318(23):2344-2345. https://doi:10.1001/jama.2017.18196

4. Buggey J., ElAmm C.A. Myocarditis and cardiomyopathy. Curr Opin Cardiol. 2018 May;33(3):341-346.

https://doi.org/10.1097/HCO.0000000000000514

5. Calus L. et al. Twelve-year follow-up study after endoscopic sinus surgery in patients with chronic rhinosinusitis with nasal polyposis //Clinical and Translational Allergy. - 2019. - ?. 9. - ?. 1. - ?. 30. https://doi.org/10.1186/s13601-019-0269-4 
6. Carter A., Dattani N., Hannan S. A. Chronic rhinosinusitis //BMJ. - 2019. - ?. 364. - ?. 1131 https://doi.org/10.1136/bmj.1131

7. Cattano D., Citardi M. J. Chronic rhinosinusitis with nasal polyps //Journal of Head and Neck Anesthesia. 2019. - ?. 3. - ? $\quad 3$. - ? $\quad$ e 20. https://doi:10.1097/HN9.0000000000000020

8. Chandy Z., Ference E., Lee J. T. Clinical Guidelines on Chronic Rhinosinusitis in Children //Current allergy and asthma reports. - 2019. - ?. 19. - ?. 2. - ?. 14 https://doi.org/10.1007/s11882-019-0845-7

9. Deosthale N.V., Khadakkar S.P., Harkare V.V., Dhoke P.R., Dhote K.S., Soni A.J., Katke A.B. Diagnostic Accuracy of Nasal Endoscopy as Compared to Computed Tomography in Chronic Rhinosinusitis. Indian J Otolaryngol Head Neck Surg. 2017 Dec;69(4):494-499. https://doi.org/10.1007/s12070-017-1232-0

10. Drago L., Pignataro L., Torretta S. Microbiological aspects of acute and chronic pediatric rhinosinusitis //Journal of clinical medicine. - 2019. - ?. 8. - ?. 2. - ?. 149. https://doi.org/10.3390/jcm8020149

11. Ghogomu N., Kern R. Chronic rhinosinusitis: the rationale for current treatments. Expert Rev Clin Immunol. $2017 \quad$ Mar;13(3):259-270. https://doi.org/10.1080/1744666X.2016.1220833

12. Grayson J. W., Harvey R. J. Topical corticosteroid irrigations in chronic rhinosinusitis //International forum of allergy \& rhinology. - 2019. - ?. 9. - ?. S1. - ?. S9-S15. https://doi.org/10.1002/alr.22331

13. Ho J. et al. Atopy in chronic rhinosinusitis: impact on quality of life outcomes //International forum of allergy \& rhinology. - 2019. - ?. 9. - ?. 5. - ?. 501-507. https://doi.org/10.1002/alr.22272

14. Hong P., Pereyra C.A., Guo U., Breslin A., Melville L. Evaluating Complications of Chronic Sinusitis. Case Rep Emerg Med. 2017; 8743828 . https://doi.org/10.1155/2017/8743828

15. Hopkins C. Chronic rhinosinusitis with nasal polyps //New England Journal of Medicine. - 2019. - ?. 381. - ?. 1. - ?. 55-63. https://doi/full/10.1056/NEJMcp1800215

16. Kristjansson R. P. et al. A loss-of-function variant in ALOX15 protects against nasal polyps and chronic rhinosinusitis //Nature genetics. - 2019. - ?. 51. - ?. 2. - ?. 267. https://doi.org/10.1038/s41588-018-0314-6

17. Marcus S. et al. The relationship between allergy and chronic rhinosinusitis //Laryngoscope Investigative Otolaryngology. - 2019. - ?. 4. - ?. 1. - ?. 13-17. https://doi.org/10.1002/lio2.236

18. Mertz L. E., Divekar R., Rank M. A. Managing Frontal Sinusitis from Systemic Inflammatory Disease //Frontal Sinus Surgery. - Springer, Cham, 2019. - ?. 273-310. https://doi.org/10.1007/978-3-319-97022-6 19

19. Pandrangi V., Reiter E. R. Impact of Sinus Surgery on Hospital Utilization for Complications of Sinusitis //Ear, Nose \& Throat Journal. - 2019. - ?. 0145561319853786. https://doi.org/10.1177\%2F0145561319853786

20. Raheja A. Endoscopic sinus surgery in sinusitis-induced intracranial abscess: concepts and controversies //Neurosurgical focus. - 2019. - ?. 47. - ?. 2. - ?. E13. https://doi.org/10.3171/2019.5.FOCUS19374

21. Rom D. et al. The Association Between Disease Severity and Microbiome in Chronic Rhinosinusitis //The Laryngoscope. - 2019. - ?. 129. - ?. 6. - ?. 1265-1273. https://doi.org/10.1002/lary.27726

22. Seo M. Y. et al. Clinical pattern of fungal balls in the paranasal sinuses: our experience with 70 patients //European Archives of Oto-Rhino-Laryngology. - 2019. - ?. 276. - ?. 4. - - ?. 1035-1038. https://doi.org/10.1007/s00405-018-5258-z

23. Wu J., Jain R., Douglas R. Effect of paranasal anatomical variants on outcomes in patients with limited and diffuse chronic rhinosinusitis. Auris Nasus Larynx. 2017 Aug;44(4):417-421.

https://doi.org/10.1016/j.anl.2016.08.009

24. Yang S. F., Patel C. R., Stankiewicz J. A. Complications of Frontal Sinusitis //Frontal Sinus Surgery. - Springer, Cham, 2019. - ?. 329-338. https://doi.org/10.1007/978-3319-97022-6_22

25. Zhang L., Zhang L.H. Comparison of different endoscopic scoring systems in patients with chronic rhinosinusitis: reliability, validity, responsiveness and correlation. Rhinology. 2017 Dec 1;55(4):363-368. https://doi.org/10.4193/Rhin17.109. 